\title{
Quantitative Schlieren Image-Noise Reduction Using Inverse Process and Multi-Path Integration
}

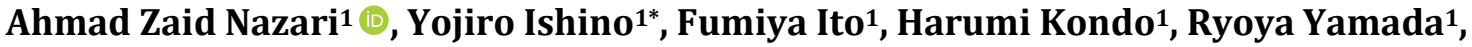 \\ Takanori Motohiro1, Yu Saiki', Yoshiaki Miyazato², Shinichiro Nakao² \\ ${ }^{1}$ Electrical and Mechanical Engineering Department, Nagoya Institute of Technology, Nagoya, Japan \\ ${ }^{2}$ Department of Mechanical Systems Engineering, The University of Kitakyushu, Kitakyushu, Japan \\ Email: *ishino@nitech.ac.jp
}

How to cite this paper: Nazari, A.Z., Ishino, Y., Ito, F., Kondo, H., Yamada, R., Motohiro, T., Saiki, Y., Miyazato, Y. and Nakao, S. (2020) Quantitative Schlieren Image-Noise Reduction Using Inverse Process and Multi-Path Integration. Journal of Flow Control, Measurement \& Visualization, 8, 25-44. https://doi.org/10.4236/jfcmv.2020.82002

Received: October 13, 2019

Accepted: March 4, 2020

Published: April 30, 2020

Copyright $\odot 2020$ by author(s) and Scientific Research Publishing Inc. This work is licensed under the Creative Commons Attribution International License (CC BY 4.0).

http://creativecommons.org/licenses/by/4.0/

(c) (i) Open Access

\begin{abstract}
This report deals with introducing two new techniques based on a novel concept of complex brightness gradient in quantitative schlieren images, "inverse process" and "multi-path integration" for image-noise reduction. Noise in schlieren images affects the projections (density thickness) images of computerized tomography (CT). One spot noise in the schlieren image appears in a line shape in the density thickness image. Noise effect like an infectious disease spreads from a noisy pixel to the next pixel in the direction of single-path integration. On the one hand, the noise in the schlieren image reduces the quality of the image and quantitative analysis and is undesirable; on the other it is unavoidable. Therefore, the importance of proper noise reduction techniques seems essential and tangible. In the present report, a novel technique "multi-path integration" is proposed for noise reduction in projections images of CT. Multi-path integration is required the schlieren brightness gradient in two orthogonal directions. The 20-directional quantitative schlieren optical system presents only images of schlieren brightness in the horizontal gradient and another 20-directional optical system seems necessary to obtain vertical schlieren brightness gradient, simultaneously. Using the "inverse process", a new technique enables us to obtain vertical schlieren brightness gradient from horizontal experimental data without the necessity of a new optical system and can be used for obtaining any optional directions of schlieren brightness gradient.
\end{abstract}

\section{Keywords}

Noise Reduction Technique, Inverse Process, Quantitative Schlieren Optical System, Complex Schlieren Brightness, Computerized Tomography (CT) 


\section{Introduction}

Schlieren imaging technique is a common tool in science and technology to visualize density gradients and investigate phenomena with non-uniform density flows in transparent media. Recently, this technique is reviewed in [1] and is shown that schlieren photography has been developed from a qualitative visualization method into a quantitative measurement method. In previous works [2]-[7], by employing and developing a non-scanning three-dimensional computerized tomography (3D-CT) technique using a delicate multi (20)-directional quantitative schlieren optical system with a flashlight source, measurement of the instantaneous density distributions of several types of flames in laminar and turbulent flows [2] [3] [4] [5] [6] and supersonic micro-jets [7] have been successfully obtained.

In the previous work [2], the target high-speed turbulent burner flames are investigated and reported. Figure 1 depicts the target flame, details dimensions of burner nozzle, coordinate system and 3D bird's-eye view of the CT-reconstructed density data. For more information related to the flow conditions, measured parameters of target flames and burner details refer to the previous work [2].

In the present report, two new techniques are introduced based on a novel concept of complex brightness gradient in the quantitative schlieren images, "inverse process" and "multi-path integration" for image-noise reduction. The new techniques partially are presented in international conferences [8] [9], in detail and entirely will be discussed here. Furthermore, it will be shown an important ability of complex schlieren brightness gradient, which is independence on the path and starting point in the integration process.

The schlieren images brightness change gradually (brightness gradient), while

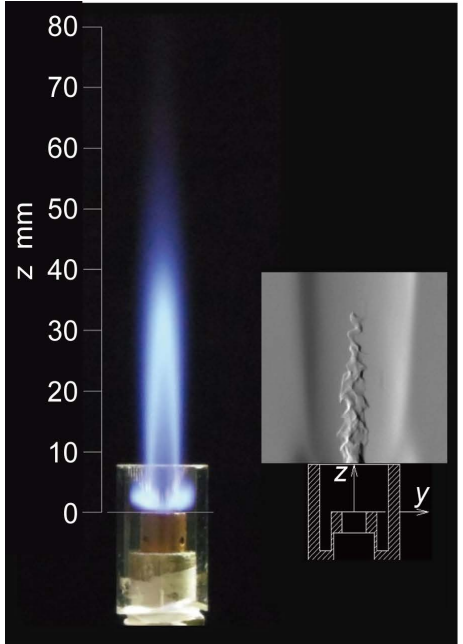

(a)

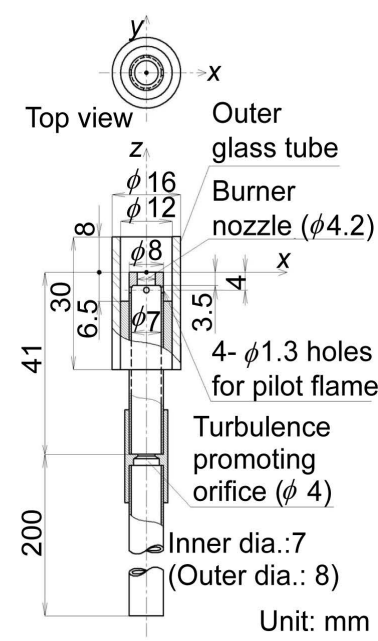

(b)

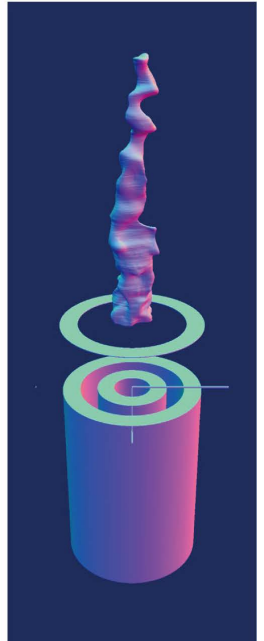

(c)

Figure 1. High-speed turbulent flame, burner, coordinate system and bird's-eye view of 3D model. (a) Target flame \& schlieren image; (b) Burner tip details; (c) 3D model view. 
image noise is a random variation of brightness and is generally considered undesirable. Noise can deduct the quality of the image and quantitative analysis [10]. Noise in the schlieren images can arise from density gradients anywhere between the source slit and the knife-edge in the wind-tunnel research, and that is why a multiple-source schlieren system is introduced for noise reduction in [11]. Quantitative schlieren imaging requires low noise level images accordingly high data accuracy. Noise in the schlieren imaging is unavoidable. There are very few noise reduction techniques studies in the works of literature. Therefore, the lack of proper noise reduction techniques is clear. Some image noise generating sources are as follows: dusty photography surroundings, dusty and dirty lenses, defective lens surface, digital cameras, and lenses. In the present study, a new approach called "multi-path integration" novel technique against former technique "single-path integration" is introduced for noise reduction in projections (density thickness) images of computerized tomography (CT).The new multi-path integration technique is required schlieren brightness gradients in both horizontal ( $x$-directional) and vertical ( $z$-directional) directions, or in other words, in two orthogonal directions (e.g. horizontal and vertical or two perpendicular diagonal directions). The 20-directional quantitative schlieren optical system gives only images of schlieren brightness in the horizontal gradient ( $x$-directional) and another 20-directional optical system seems necessary to obtain vertical ( $z$-directional) schlieren brightness gradient, simultaneously. The present investigation represents the new "inverse process" novel technique for obtaining vertical and diagonal schlieren brightness gradient from horizontal experimental data (with vertical knife-edge orientation) without the necessity of a new optical system. The main advantages of the new approach employed are its relative simplicity, the ease of application, without extra cost, and the satisfactory accuracy of results and can be used for obtaining any optional direction of schlieren brightness gradients. Recently [8], we presented the "inverse process" technique but in a different way than this time. In the previous work, the "inverse process" was performed after the CT-reconstruction, however, now is done before the CT-reconstruction procedure (Figure 2). Indeed, in the previous work phantom (virtual) data of schlieren images are obtained by the "inverse process" and used to evaluate noise reduction technique while in the new "inverse process" technique actual experimental data is used for reproducing other directions of schlieren brightness gradient.

By using both white-light and monochromatic sources, color schlieren images have been produced from combining horizontal and vertical gradient brightness of common schlieren images by [12], but for this purpose, two independent knife-edges along two different beam paths are employed. About 70 years ago, a more complex arrangement is presented for obtaining two spatially separated images sensitive to orthogonal path deviations [13]. A good example of the same numerical supersonic jet-exit flow field is rendered as shadowgraph, bright-field schlieren (circular cutoff), vertical and horizontal knife-edge schlieren in [14]. 


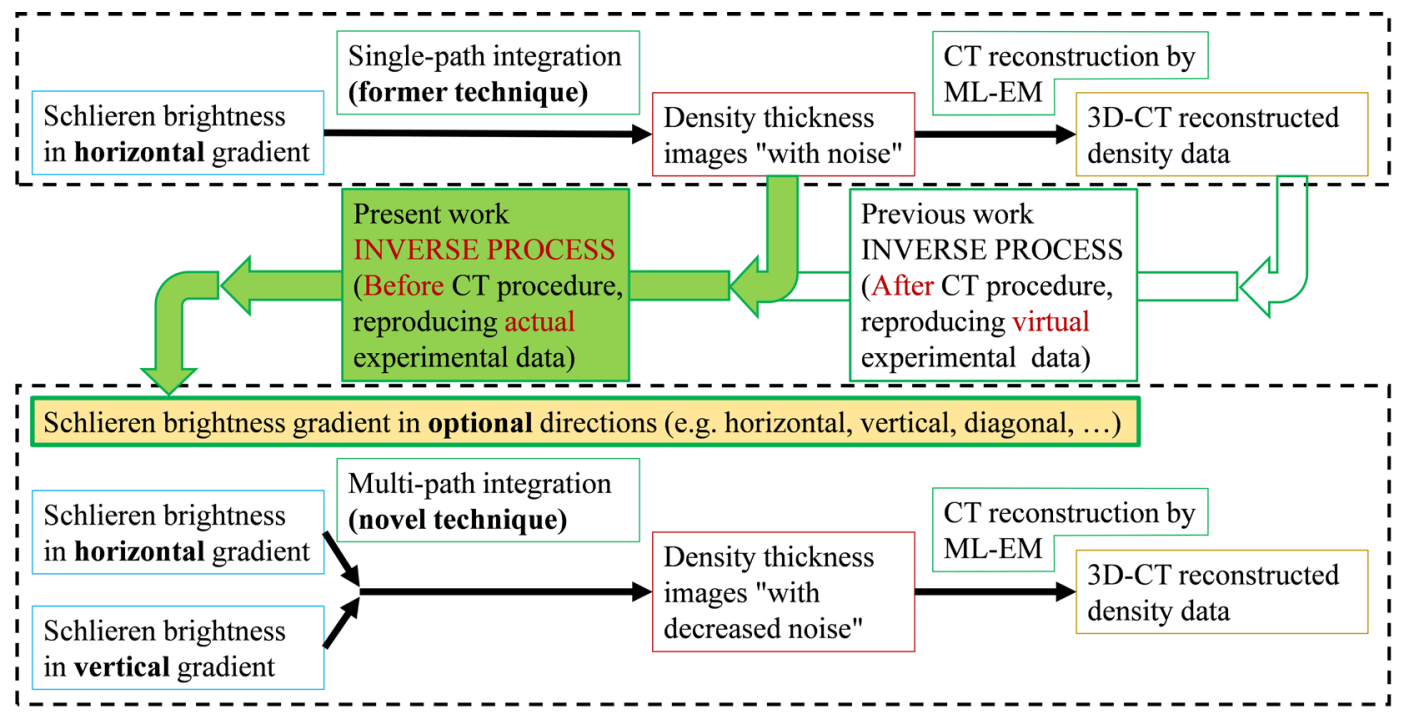

Figure 2. Flow diagram of the "inverse process" new technique for the reproduction of schlieren brightness gradient in horizontal, vertical, diagonal directions and conversion to the new projections (density thickness) of CT by new "multi-path integration" technique.

In the current investigation, the horizontal schlieren brightness gradient data of high-speed turbulent flame [2] with average flow velocity $10 \mathrm{~m} / \mathrm{s}$ is used for reproducing the 20-directional images of schlieren brightness in horizontal, vertical and diagonal gradients.

First, as shown in Figure 2 (top), the initial density thickness ( $D t$ ) images are obtained from images of schlieren brightness in the horizontal gradient ( $x$-directional). Next, using the inverse process from density thickness $(D t)$ images, the horizontal ( $x$-directional), vertical ( $z$-directional) and two diagonals $\left(45^{\circ}\right.$ and $\left.135^{\circ}\right)$ schlieren brightness gradient data of target flame have been successfully obtained. Finally, by applying the multi-path integration technique, the new set of projections images of density thickness are successfully obtained (Figure 2 (bottom)). Then, by using the new set of density thickness images "with decreased noise" as projections for CT-reconstruction the 3D density distributions of target flame have been successfully reconstructed.

\section{Experimental Apparatus Setup and Methods}

\subsection{Multi-Directional Quantitative Schlieren Optical System}

Figures 3(a)-(c) illustrates the concept of a 20-directional schlieren camera. In the camera system, the target flame/non-uniform density field is observed from $180^{\circ}$ direction using numerous schlieren optical systems simultaneously from $\theta$ $=-85.5^{\circ}$ to $+85.5^{\circ}$ at an interval of $9^{\circ}$. Here, angle $\theta$ is defined as the horizontal angle from the $x$-axis. For pre-investigation and for time series observation (high-speed Schlieren movies) of the target flow, a high-speed camera (HSC) is used simultaneously with 20-direction Schlieren photographing apparatus (between cameras No. 13 and No. 14, Figure 3(b)). 


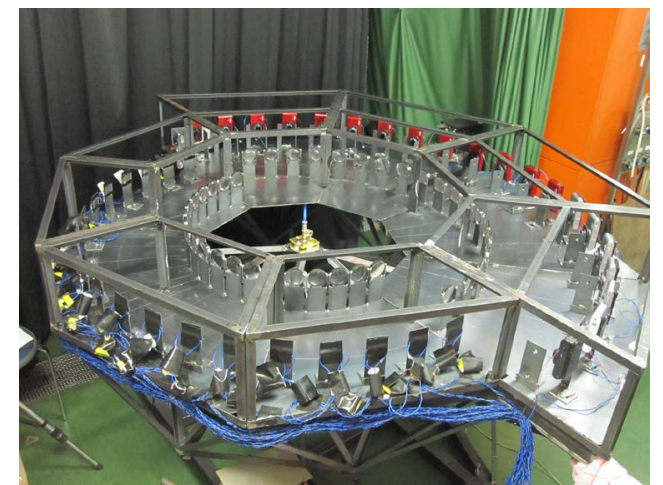

(a)

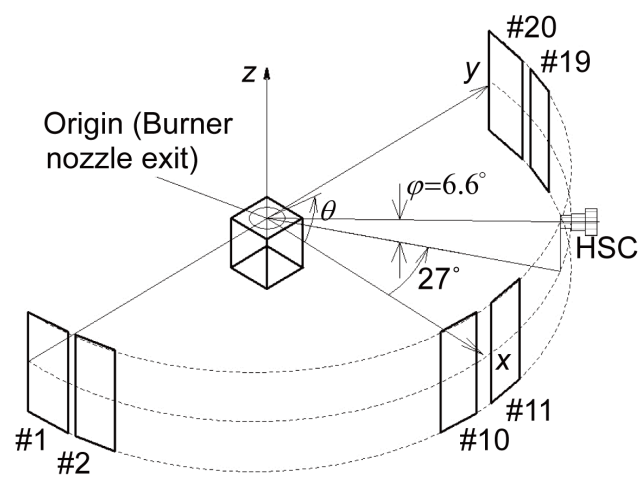

(b)

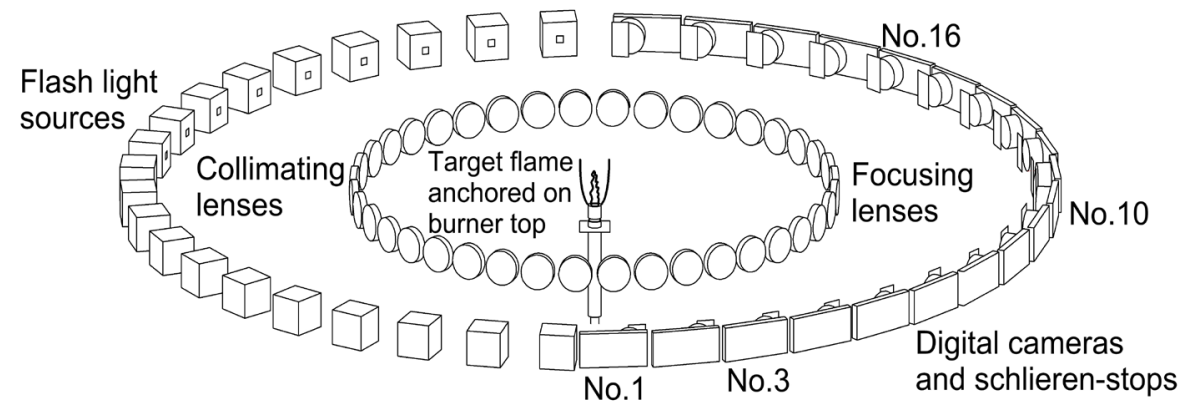

(c)

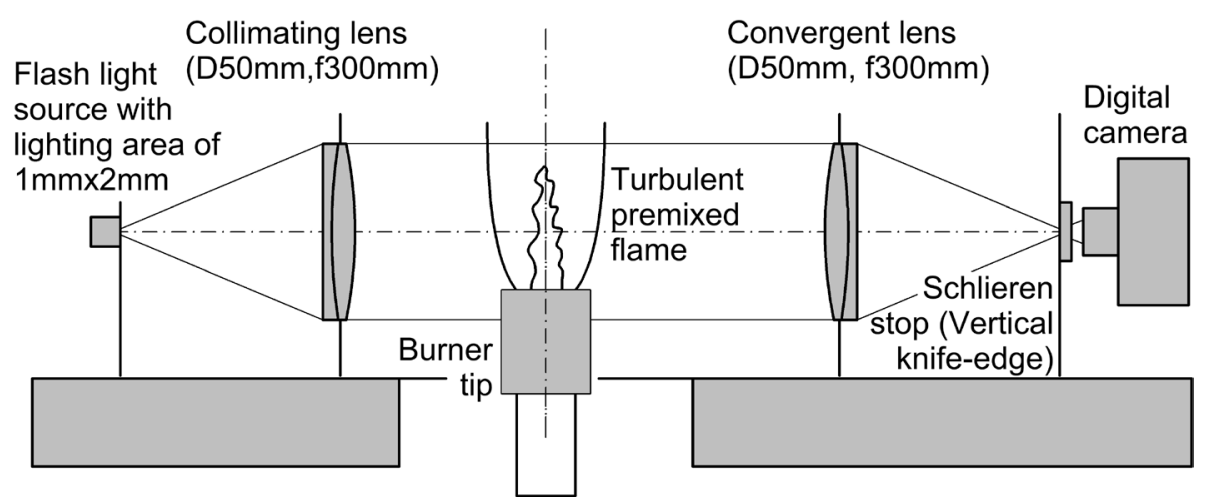

(d)

Figure 3. Multi-direction schlieren optical system and coordinate system [2]-[9]. (a) Schlieren camera system; (b) Coordinate system; (c) Schematic of 20-direction schlieren camera; (d) Single unit of quantitative schlieren optical system.

The diagram of a single instance of the multi-directional quantitative schlieren camera system is depicted in Figure 3(d). This system is composed of two convex achromatic lenses of $50 \mathrm{~mm}$ diameter and $300 \mathrm{~mm}$ focal length, a flashlight source unit, a vertical knife-edge (for obtaining horizontal schlieren brightness gradient images), and digital camera.

The neutral-density (ND) filter (Fuji 1.5, exposure adjustment multiple 3.16) and stepped neutral-density filter is used for calibrating the cameras. The light unit is a xenon flashtube that emits full-spectrum white light with a uniform luminance rectangular area of $1 \mathrm{~mm} \times 2 \mathrm{~mm}$ and a $35 \mu$ s duration. 


\subsection{Image Processing}

Figure 4 shows a conceptual framework for the formation of a projection image (density thickness) in the proposed schlieren 3D-CT system. Detailed descriptions of the various sections of Figures 4(a)-(k), along with the supporting formula, are given in the previous reports [2]-[9] and many other references cited therein. Here, for brevity, the main points of Figure 4 are explained as follows. The first goal is obtaining the density thickness of deviation density $\left(D t^{\star}(X(\theta))\right)$ which shown in Figure 4(d) with a unit of $\left(\mathrm{kg} / \mathrm{m}^{3}\right)(\mathrm{m})$. The density thickness of deviation density $D t^{\star}(X(\theta))$ is obtained automatically from schlieren observation using spatial integration of deviation density $\Delta \rho^{\star}(X(\theta), Y)$ along the line of sight. In practice for obtaining density thickness of deviation density (Figure 4(i)), the image processing activity starts from Figure 4(e) by obtaining two sets of images, "with target" and "without target" (without any disturbance in the test section) with a horizontal brightness gradient in the $X$-direction. Two sets of images present by schlieren observation as $B(X)$ and $B_{n f}(X)$ (brightness of schlieren


Figure 4. Processes of formation of schlieren brightness and conversion to projections (density thickness $(D t)$ ) of CT. 
image in no-flame condition). To obtain the density thickness of deviation density $D t(X(\theta))$ from $B(X)$ and $B_{n f}(X)$, both are processed in the following manner of Figure 4(f)-(i). As indicated in Figure 4(f) and Figure 4(g), deviation brightness in schlieren image $\Delta B(X)$

$$
\Delta B=B(X)-B_{n f}(X)
$$

is scaled to $\mathrm{d}(D t) / \mathrm{d} X$ by

$$
\mathrm{d}(D t) / \mathrm{d} X=-(1 / K)(\Delta s / f)\left[\Delta B(X) / B_{n f}(X)\right]
$$

where $K$ is Gladstone-Dale constant for air $\left(K=2.26 \times 10^{-4} \mathrm{~m}^{3} / \mathrm{kg}\right), \Delta s(=1 \mathrm{~mm}$ $\times 2 \mathrm{~mm}$ (Hor. $\times$ Ver.)) is the transparent width of the light source image on schlieren stop location and $f(=300 \mathrm{~mm})$ is the focal length of the convergent lens. Deviation density thickness $D t(X(\theta))$ is, therefore, reproduced by transverse integration of $\mathrm{d}(D t) / \mathrm{d} X$ from schlieren images, as shown in Figure $4(\mathrm{~h})$. Finally, the "density thickness" $D t^{\prime}(X(\theta))$ is obtained by adding the thickness of $\left(\rho_{\text {ref }}-\rho_{a}^{*}\right)$ to the deviation density thickness $D t(X(\theta))$ on peripheral of the observed range of radius $R$ (Figure 4(i)), as expressed by

$$
D t^{\prime}(X(\theta))=D t(X(\theta))+2\left(R^{2}-X^{2}(\theta)\right)^{1 / 2}\left(\rho_{r e f}-\rho_{a}^{*}\right)
$$

In the present study, $\rho_{\text {ref }}=\rho_{a}^{*}$, accordingly, $D t^{\prime}(X(\theta))=D t(X(\theta))$.

\subsection{CT-Reconstruction}

Density thickness images are used for CT-reconstruction by maximum likelihood-expectation maximization (ML-EM) [15] an appropriate CT algorithm to obtain the 3D reconstruction of density distribution, the ML-EM method [2]-[9] is employed for CT-reconstruction. The CT procedure is carried out in each horizontal plane of $z$-axis for the reconstruction of deviation density distribution $\Delta \rho(x, y)$ from a linear data set of density thickness $D t^{\prime}(X(\theta))$ (Figure $4(\mathrm{j})$ ). Finally, 2D density distribution (Figure $4(\mathrm{k})$ ) is obtained as follow.

$$
\rho(x, y)=\rho_{a}^{*}-\Delta \rho(x, y)
$$

The reconstruction was performed cross-section by cross-section and then the cross-sections were stacked to form a three-dimensional density distribution. Therefore, a 2D distribution $\rho(x, y)$ is accumulated in layers to form the 3D-CT distribution $\rho(x, y, Z)$. In the present study, the "density thickness" projections images of $400(H) \times 500(V)$ pixel $(32 \mathrm{~mm} \times 40 \mathrm{~mm})$ are used for CT-reconstruction to produce 3D data $400(x) \times 400(y) \times 500(z)$ pixel $(32 \mathrm{~mm}$ $\times 32 \mathrm{~mm} \times 40 \mathrm{~mm}$ ). The voxel size is $0.08 \mathrm{~mm}$ in each direction.

\section{New Techniques and Treatments}

\subsection{Inverse Process}

As mentioned before, in the present study, using "inverse process" new approach from density thickness (Dt) images (Figure 2), the horizontal 
$\left(x\right.$-directional), vertical ( $z$-directional) and two diagonals $\left(45^{\circ}\right.$ and $\left.135^{\circ}\right)$ schlieren brightness gradient data of target flame have been successfully obtained. The relation between deviation brightness $\Delta B$ and density thickness $D t$ is expressed by Equation (2). Equation (2) can be rewritten in the following simple forms.

$$
\mathrm{d}(D t) / \mathrm{d} X \propto \Delta B(X)
$$

and

$$
\mathrm{d}(D t) / \mathrm{d} X=\alpha \times \Delta B(X)
$$

where $\alpha$ is a coefficient with parameters that explained in Equation (2). The deviation density thickness can be expressed as,

$$
\mathrm{d}(D t(x, z)) / \mathrm{d} X=D t(x, z)-D t(x-1, z)
$$

Finally, in the inverse process, by combining the Equation (6) and (7), the deviation brightness of the quantitative schlieren image in each direction will be given by:

$$
\begin{aligned}
& \Delta B(x, z)_{\text {Horizontal },+x}=(1 / \alpha) \times[D t(x, z)-D t(x-1, z)] \\
& \Delta B(x, z)_{\text {Vertical },+z}=(1 / \alpha) \times[D t(x, z)-D t(x, z-1)] \\
& \Delta B(x, z)_{\text {Diagonal }, 45^{\circ}}=(1 / \alpha) \times[D t(x, z)-D t(x-1, z-1)] \\
& \Delta B(x, z)_{\text {Diagonal }, 135^{\circ}}=(1 / \alpha) \times[D t(x, z)-D t(x+1, z-1)]
\end{aligned}
$$

The direction of the schlieren brightness gradient can be changed in the opposite direction (Figures $5(\mathrm{c} 2)$-(f2)) only by applying one minus sign in the corresponding equation. Therefore, by using density thickness $D t$ in the inverse process, the schlieren brightness in horizontal, vertical and diagonal directions can be calculated (Equation (8)-(11)) without the necessity of a new optical system.

One of the benefits of using this approach is that now we can apply the "multi-path integration" noise reduction technique. The sample results for only camera number 10 are depicted for all mentioned directions in Figure 5. The corresponding result for numerical simulation (refer to section 3.4) of the flame is depicted in the top right side and the corresponding knife-edge orientation is shown underneath of each schlieren image in Figure 5.

A key element in the schlieren setup is the knife-edge. In traditional schlieren imaging, gradients in the refractive index normal to the imaging plane are viewed by focusing the deflected light rays onto a knife-edge. The rays that are deflected above the knife-edge appear as bright regions in the image while rays that are deflected onto the knife-edge appear as dark regions in the image. The knife-edge serves as a cut-off filter for light intensity (brightness). The knife-edge orientation will reveal directional density gradients (brightness gradients). For example, vertical knife-edge orientation present horizontal 


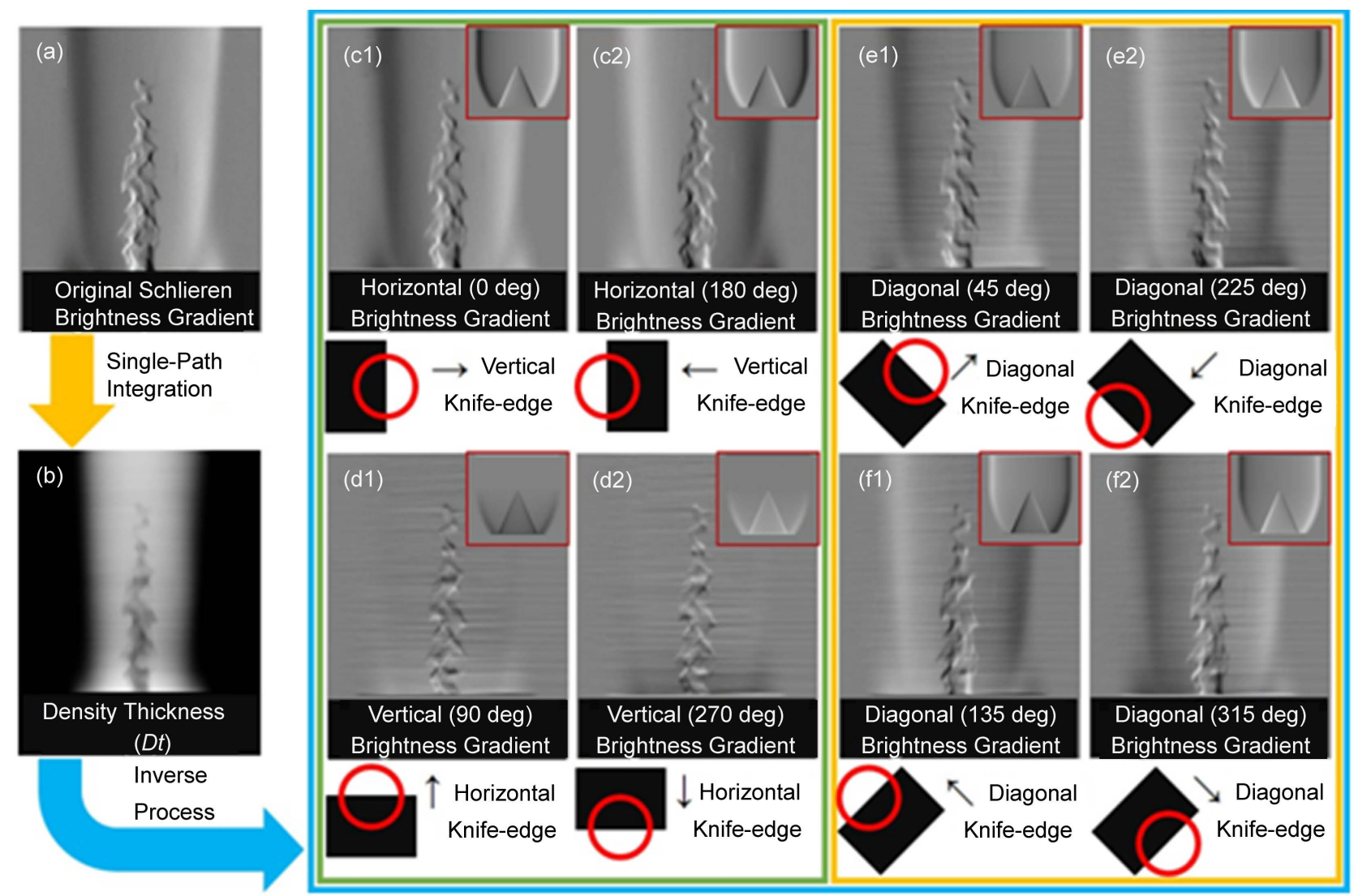

Figure 5. Inverse process for reproduction of schlieren brightness in horizontal, vertical and diagonal gradient.

schlieren brightness gradient data and horizontal knife-edge orientation present vertical schlieren brightness gradient data. In the 20-directional quantitative schlieren optical system, a vertical knife-edge (Figure $5(c 1)$ ) is used which for adjusting moves from left to right in the positive $x$-direction.

\subsection{Complex Schlieren Brightness Gradient}

Figure 6 shows an example of contour lines display of density deviation thickness (density thickness). At this figure, the brightness value of the Schlieren image is given by the gradient (slope) of the curved surface. For example, when a knife-edge parallel to the $z$-axis is used as a schlieren stop (vertical knife-edge) and the light beam is cut in the $+x$ direction, the gradient (slope) of the red curve can be obtained by measuring the brightness of the schlieren image. On the other hand, if the knife-edge is inclined $90^{\circ}$, the slope of the blue curve can be obtained.

Therefore, by simultaneously considering the schlieren brightness gradient information in the horizontal direction (red, real part) and vertical direction (blue, imaginary part), the complex gradient of the density thickness distribution at any position can be obtained. The spatial integration to obtain the density thickness distributions can now be performed on any arbitrary path, and noise reduction now becomes possible. In this study, a schlieren image 


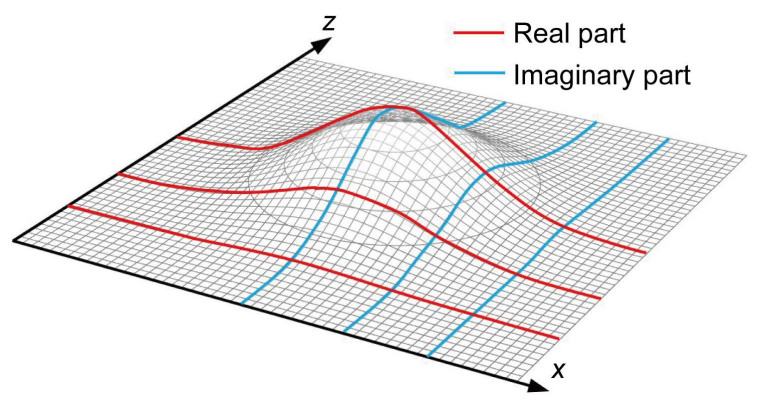

Figure 6. Complex schlieren image explanation.

with information on the gradient of density thickness in two orthogonal directions is called a "complex schlieren image".

\subsection{Cauchy Integration Theorem}

The Cauchy integration theorem in complex analysis is an important statement about line integrals for regular functions in the complex plane. Cauchy's integration theorem states that if $f(z)$ be a regular function in the simply connected domain $D$, its contour integral on a path does not depend on the integration path and depends only on the beginning and ending points of the path. Therefore, if let $C$ be a piecewise continuously differentiable path in $D$ with start point $a$ and end point $b$, and $F$ is a complex antiderivative of $f$, then,

$$
\int_{C} f(z)=\int_{a}^{b} f(z)=F(b)-F(a)
$$

If $a=b$, then $C$ is a closed curve (loop) within $D$, accordingly,

$$
\oint_{C} f(z)=0
$$

We applied the above theorem to develop the noise reduction technique. As shown in Figure 7 and Figure 10, for obtaining density thickness images "with decreased noise" the average of transverse integration from multi-path is employed.

It is remarkable, as a new finding, a new important concept in the complex schlieren image could be expressed as follow; The complex schlieren image for obtaining density thickness at an arbitrary position (ending point of the integration path) has one more potential power not depends on the starting point of the integration path. Therefore, the calculation of density thickness can be performed just by starting integration from any point on any path (Figure 10).

\subsection{Numerical Simulation}

First, as a preliminary investigation and for a deep understanding of complex schlieren brightness, a numerical simulation for schlieren brightness of a flame is performed. The results of the numerical simulation are depicted in Figure 7. Schlieren images in horizontal, vertical and diagonal directions of brightness gradients are shown in Figures 7(a1)-(h1). One hundred noises with various brightness in different positions are added to the schlieren images randomly. 



(a2) Single-path integration in $+x$
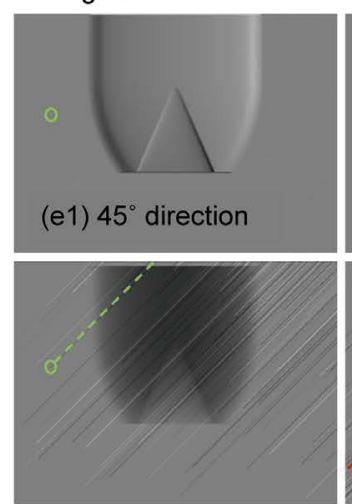

(e2) Single-path integration in $45^{\circ}$



(b2) Single-path integration in $-x$
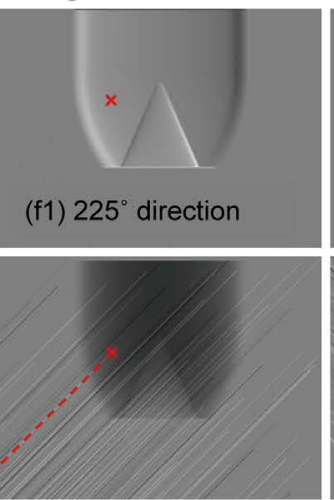

(f2) Single-path integration in $225^{\circ}$ (c1) $+z$ direction

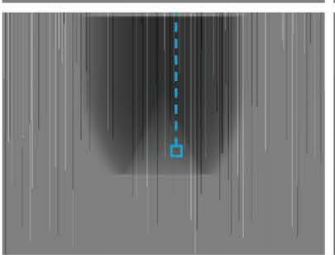

(c2) Single-path integration in $+z$
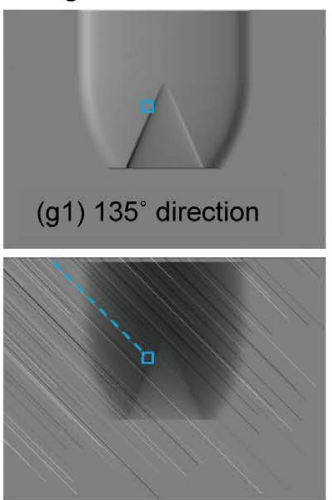

(g2) Single-path integration in 135
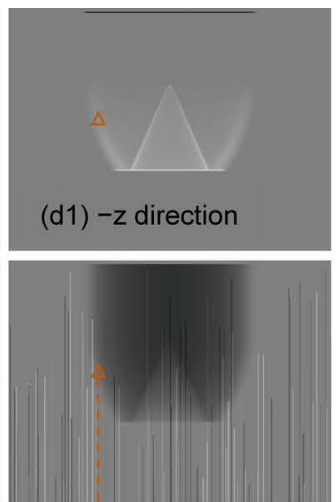

(d2) Single-path integration in $-z$
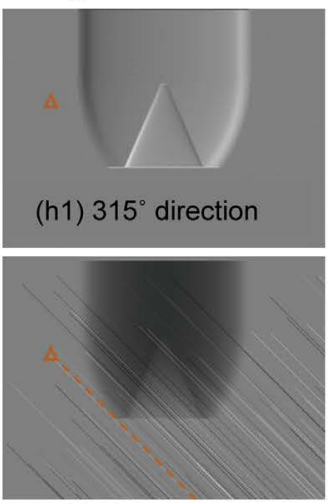

(h2) Single-path integration in $315^{\circ}$

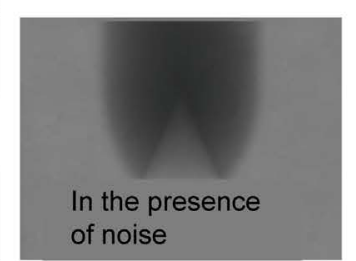

(i1) Multi-path integration

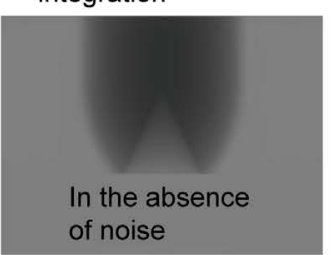

(i2) Multi-path integration

Figure 7. (a1)-(h1) Numerical simulation of schlieren brightness gradient in the horizontal, vertical and diagonal directions of flame, (a2)-(h2) Noisy density thickness ( $D t$ ) brightness using "single-path integration" underneath of the corresponding each schlieren image and (i) Density thickness brightness using "multi-path integration" with reduced noise effects.

The noisy density thickness images obtained by single-path integration (former technique) are depicted underneath of corresponding each schlieren images (Figures 7(a2)-(h2)). Figure 7(i) illustrates the density thickness image by employing the "multi-path integration" new technique. Figure 7 (i2) depicts the result of the density thickness image in the absence of noise in the schlieren image.

Figure 8 depicts a schlieren brightness gradient (Figure 8 (left)) at the yellow dashed line position for the numerical simulation result (Figure 8 (right)).The brightness of schlieren image changes gradually (brightness gradient), while image noise is the variation of brightness suddenly (Figure 8) and is generally considered undesirable. The existence of some noises in the schlieren image affects the density thickness image (Figure 9). Noise effect like an infectious disease spreads from a noisy pixel to the next pixel in the direction of single-path integration former technique (Figure $9(\mathrm{c})$ ). One spot noise in the schlieren image appears in a line shape in the density thickness image, which starts from noise position and continues as a line in the direction of single-path integration (Figure 9(c)). As seen in Figure 7(i1) and Figure 9(d), using the new technique the noise effects are reduced to an acceptable level and slightly present around 



Figure 8. Schlieren brightness gradient at the yellow dashed line position with noise effects.


Figure 9. Noise effects on the density thickness $(D t)$ brightness gradient (simulation result).

initial noise spots (this may be a disadvantage of the proposed technique).

Noise in schlieren imaging is unavoidable and can detract from the quality of the image and subsequent visual and quantitative analysis. Therefore, the need for proper noise reduction techniques is clear.

\subsection{Multi-Path Integration Technique}

As mentioned before, a new approach called "multi-path integration" novel technique against the former technique "single-path integration" is introduced for noise reduction in the projections (density thickness) images of CT. The new multi-path integration technique is required schlieren brightness gradients in both horizontal ( $x$-directional) and vertical ( $z$-directional) directions, or in other words, in two orthogonal directions (e.g. horizontal and vertical or two perpendicular diagonal directions) (Figure 2).

In the usual procedure, density thickness image reproduces by transverse integration of the schlieren image brightness in the single-path along schlieren brightness gradient (path No. 4 in Figure 10 (left)). 
In the present study, for obtaining density thickness images "with decreased noise" the average of transverse integration from multi-path is employed. These paths are in four main horizontal and vertical directions (totally 28 paths, 7 paths in positive and 7 paths in negative of $x$ and $z$ directions) (Figure 10), as well as, the paths can be used from two perpendicular diagonal directions. The new procedure "multi-path integration" is one comprehensive technique that including the usual former "single-path integration" technique as well. In the former technique "single-path integration" (path No. 4, Figure 10), the density thickness for pixel $(x, z)$ can be expressed from Equation (7) as follow.

$$
D t(x, z)=D t(x-1, z)+\mathrm{d}(D t(x, z)) / \mathrm{d} x
$$

On the right side of Equation (14), the first term is the density thickness data from prior adjacent pixel (the density thickness for the first-pixel column $(1, z)$ is assumed zero) and the second term comes from the schlieren image brightness (Equation (2)). This second term for $z$ (vertical) direction can be obtained by applying the inverse process technique. Calculation of two sample paths (No. 6 and No. 16) in the new technique "multi-path integration" is shown in the following equations.

$$
\begin{aligned}
& D t(x, z)=D t(x-1, z+2)+\frac{\mathrm{d}(D t(x, z+2))}{\mathrm{d} x}-\frac{\mathrm{d}(D t(x, z+1))}{\mathrm{d} z}-\frac{\mathrm{d}(D t(x, z))}{\mathrm{d} z} \\
& D t(x, z)=D t(x-2, z-1)+\frac{\mathrm{d}(D t(x-2, z))}{\mathrm{d} z}+\frac{\mathrm{d}(D t(x-1, z))}{\mathrm{d} x}+\frac{\mathrm{d}(D t(x, z))}{\mathrm{d} x}
\end{aligned}
$$

First, the density thickness for pixel $(x, y)$ is obtained from the multi-path (28 paths). Then, the average of all these paths is considered as the density thickness for the target pixel.

In the schlieren imaging system, brightness gradually increases (or decreases) from ambient condition (with constant brightness) to boundary condition and vice versa. This fact is effective whether schlieren photography is containing a
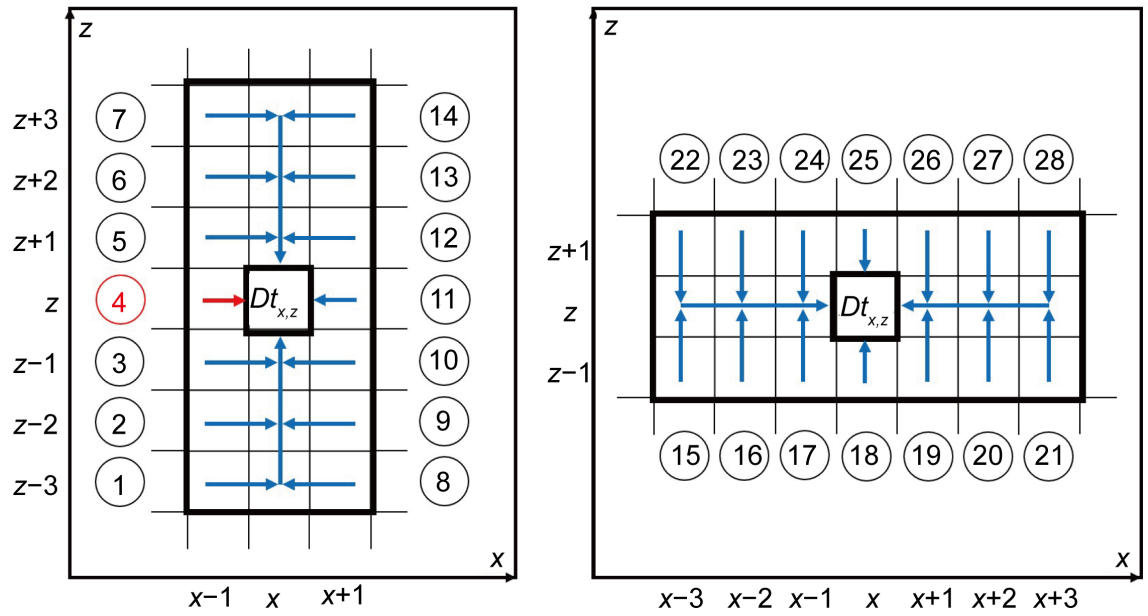

Figure 10. Integration paths (28 paths) for calculating density thickness of pixel $(x, z)$. 
complete brightness gradient in the integrating direction or not. Figure 11 represents complete (Figure 11(a)) and incomplete (Figure 11(b)) observation of the schlieren brightness gradient for the laminar flame kernel. Hence, if the schlieren photography in one and more directions is not bounded to an ambient condition which means the schlieren photography is not observed complete target flow including the boundary condition, those directions could be omitted (Figure 11(b) (Top direction)), because this treatment will give better results for noise reduction using multi-path integration. In the present study, paths from negative $z$-direction (7 paths, No. 22 to 28 , Figure 10) are not considered in the calculation due to that top area of target flame is not captured by the schlieren photography system, because of the fact that lenses diameters are smaller than target flame height.

\section{Results and Discussions}

\subsection{Quantitative Schlieren Brightness Images}

Figure 12 shows quantitative schlieren images of the horizontal brightness deviation from the original experimental data of high-speed turbulent premixed flame. The numbers inserted in each picture express the shooting angles $\theta$. The images of Figure 12 are processed by the above-mentioned "inverse process" to obtain quantitative schlieren images of vertical and diagonal brightness deviation. Due to page limitation, as a sample just for one direction for camera No.10, the results are depicted for new horizontal, vertical and diagonal (two directions) deviation brightness of quantitative schlieren images in Figure 13.

\subsection{Projections Images of Density Thickness $(D t)$}

For better evaluation of the "multi-path integration" noise reduction technique, in the new set of 20-directional schlieren images obtained from the inverse process, one thousand noises are distributed with various brightnesses and in the

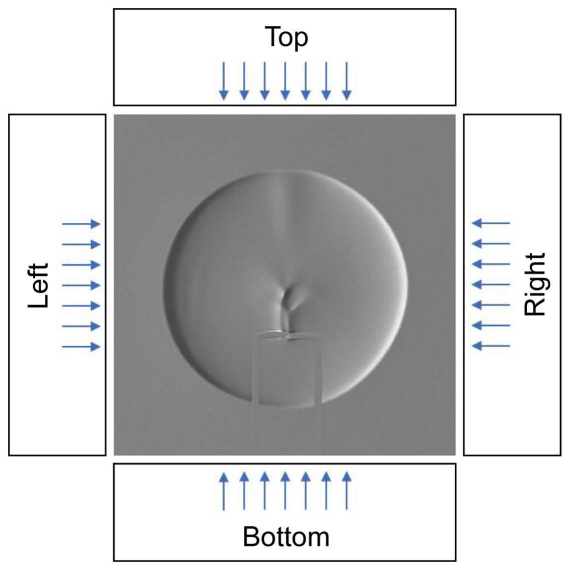

(a)

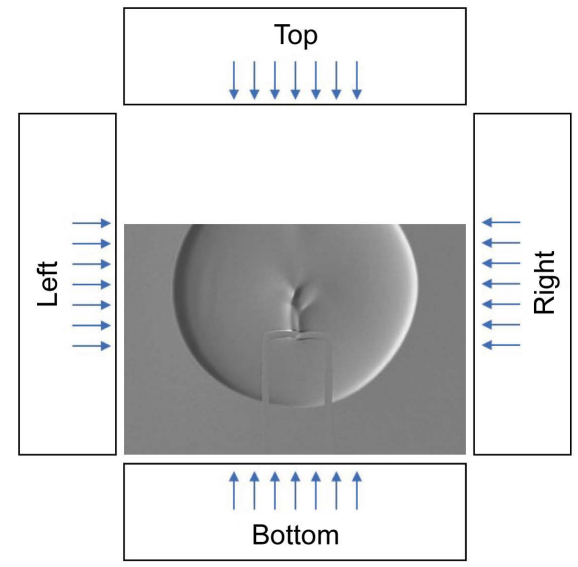

(b)

Figure 11. Sample schlieren images of the laminar flame kernel [4]. (a) Complete observation; (b) Incomplete observation. 


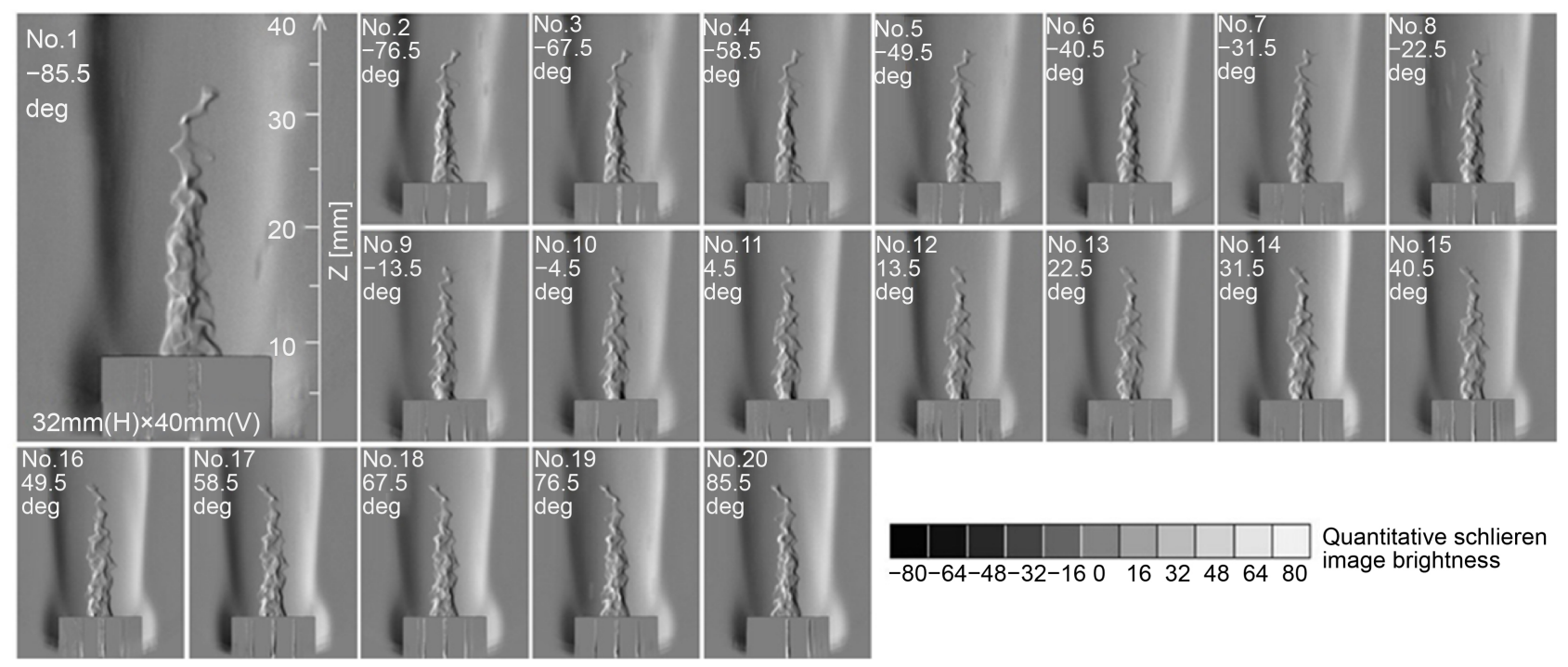

Figure 12. Original quantitative schlieren images of target flame $(U=10 \mathrm{~m} / \mathrm{s})[2]$.
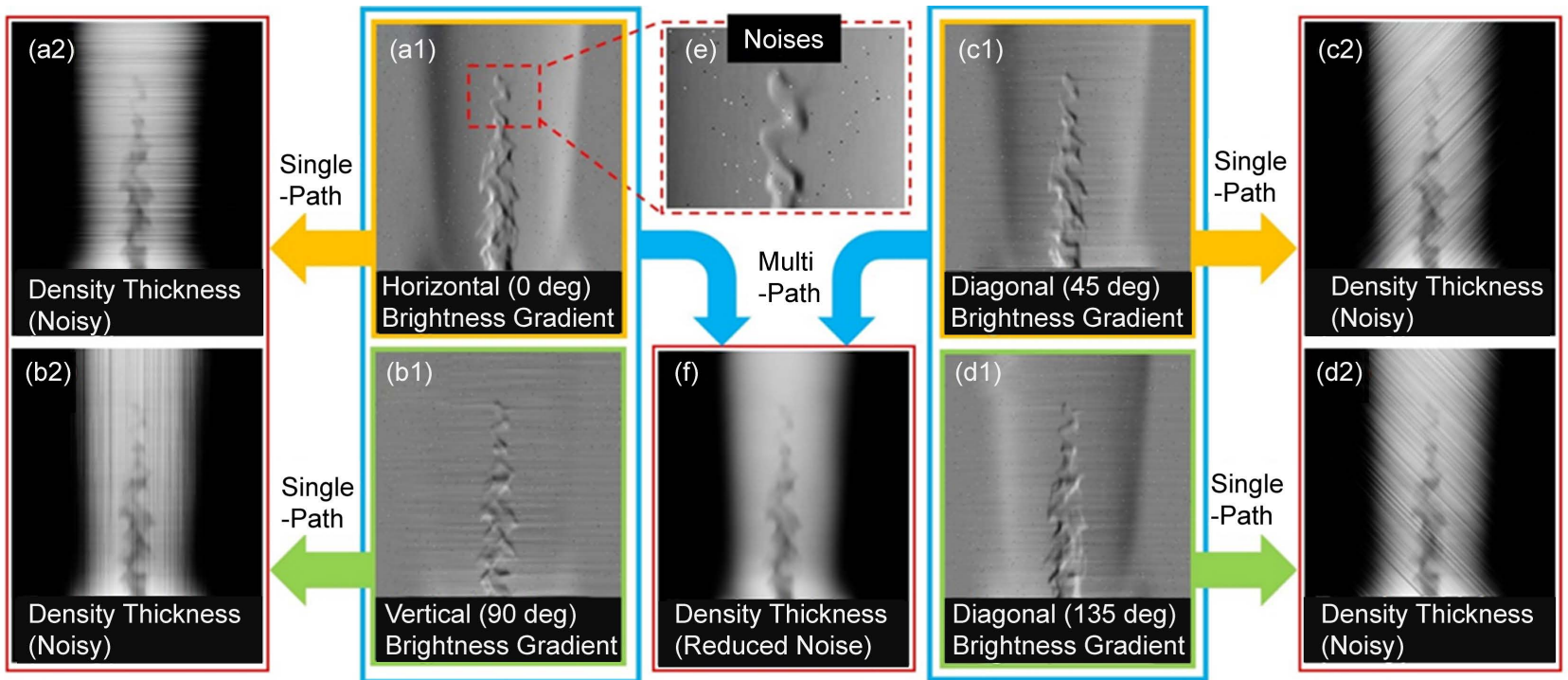

Figure 13. Schlieren brightness gradient for (a1) horizontal, (b1) vertical and (c1)-(d1) diagonals directions, (f) conversion to the new projection (density thickness $(D t)$ ) of CT by new "multi-path integration" technique, against (a2)-(d2) noisy density thickness $(D t)$ images by former "single-path integration" technique. (The schlieren images (a1-d1) and density thickness images (a2-d2) are depicted as a sample just for one direction for camera No. 10).

different positions randomly (Figure 13(e)). Then, by using the former technique "single-path integration", in horizontal (Figure 13(a1)), vertical (Figure 13(b1)) and diagonal directions (Figure 13(c1) and Figure 13(d1)), the projections images of density thickness with the existence of noise effects obtained (Figures 13(a2)-(d2)). Finally, the images of Figure 13(a1) (horizontal schlieren brightness gradient) and Figure 13(b1) (vertical schlieren brightness gradient) are processed by the above-mentioned conversion procedure "multi-path integration" to projection images of density thickness "with decreased noise level" (Figure 13(f)). It is again noteworthy that the new set of projections images of 
density thickness "with decreased noise level" can be obtained by using schlieren brightness gradient in horizontal and vertical or two perpendicular diagonal directions (Figure 13(f)). As seen in Figure 7(i1), Figure 13(f) the noise effects are reduced to an acceptable level and slightly present around initial noise spots.

\subsection{CT-Reconstruction Results}

By employing the density thickness images, noisy (Figure 13(a2)) and with decreased noise level (Figure 13(f)) as projections for CT-reconstruction, the 3D instantaneous density distributions of target flame have been successfully obtained for $z=8 \mathrm{~mm}$ to $40 \mathrm{~mm}$. Figure 14 shows a sample set of one horizontal and one vertical cross-section of 3D-CT distributions of density. The darker parts are related to lower density areas with higher temperature and the brighter parts are related to higher density points with lower temperature. For better comparing new and former techniques, the corresponding density contour diagrams of each cross-section are depicted in Figure 15, as well. The density distributions images from multi-path integration in Figure 14(c2) and Figure 14(c3) are uniform, but the results from single-path integration are streaky Figure 14(c1) and Figure 14(c4). As shown in Figure 15(c4) the density contour diagrams from single-path integration are zigzagged form in contour lines.

Figure 16 provides the quantitative information of CT-reconstructed density distributions on the line of $(y, z)=(0 \mathrm{~mm}, 24 \mathrm{~mm})$. By applying the multi-path integration technique, the density distributions diagrams in the presence of noise have coincided with the absence of noise, but the noise effects clearly

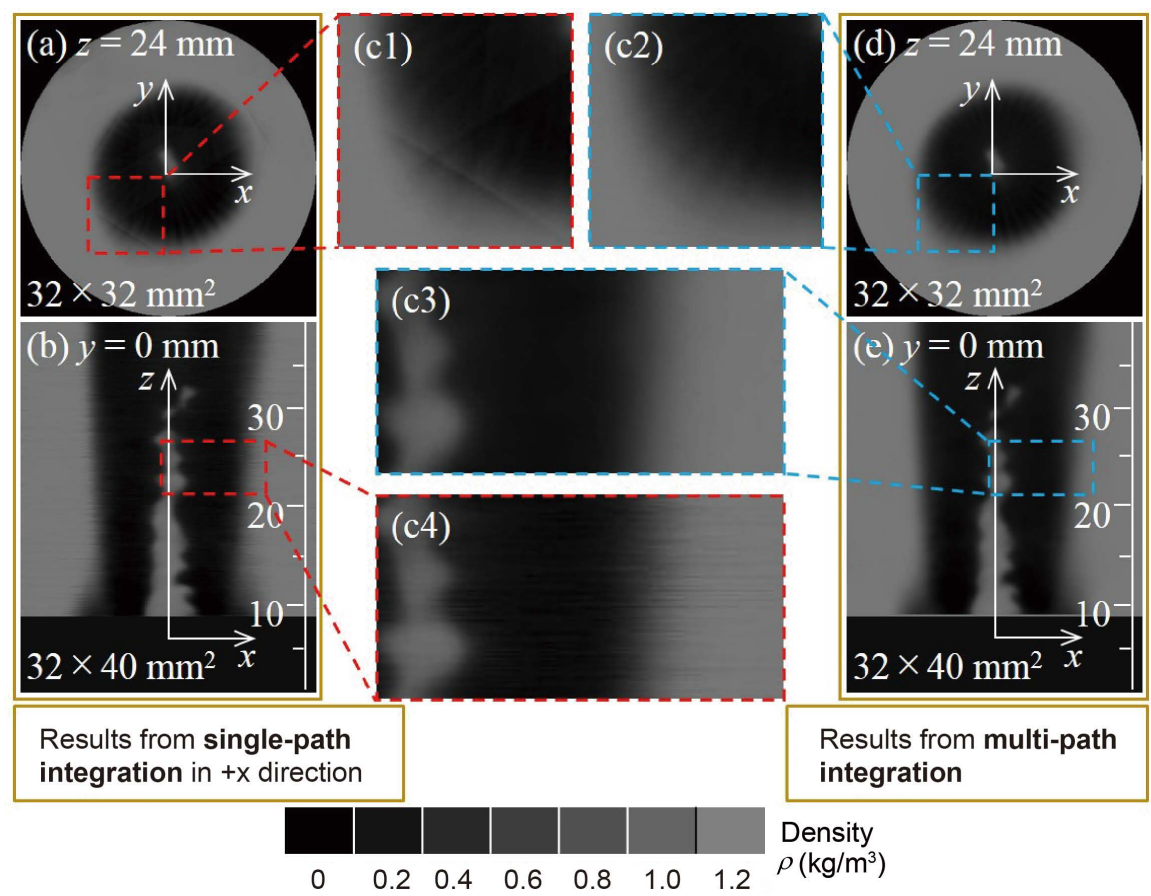

Figure 14. Sample set of horizontal ((a) and (d)) and vertical ((b) and (e)) distributions of CT-reconstructed density distributions of target flame. 


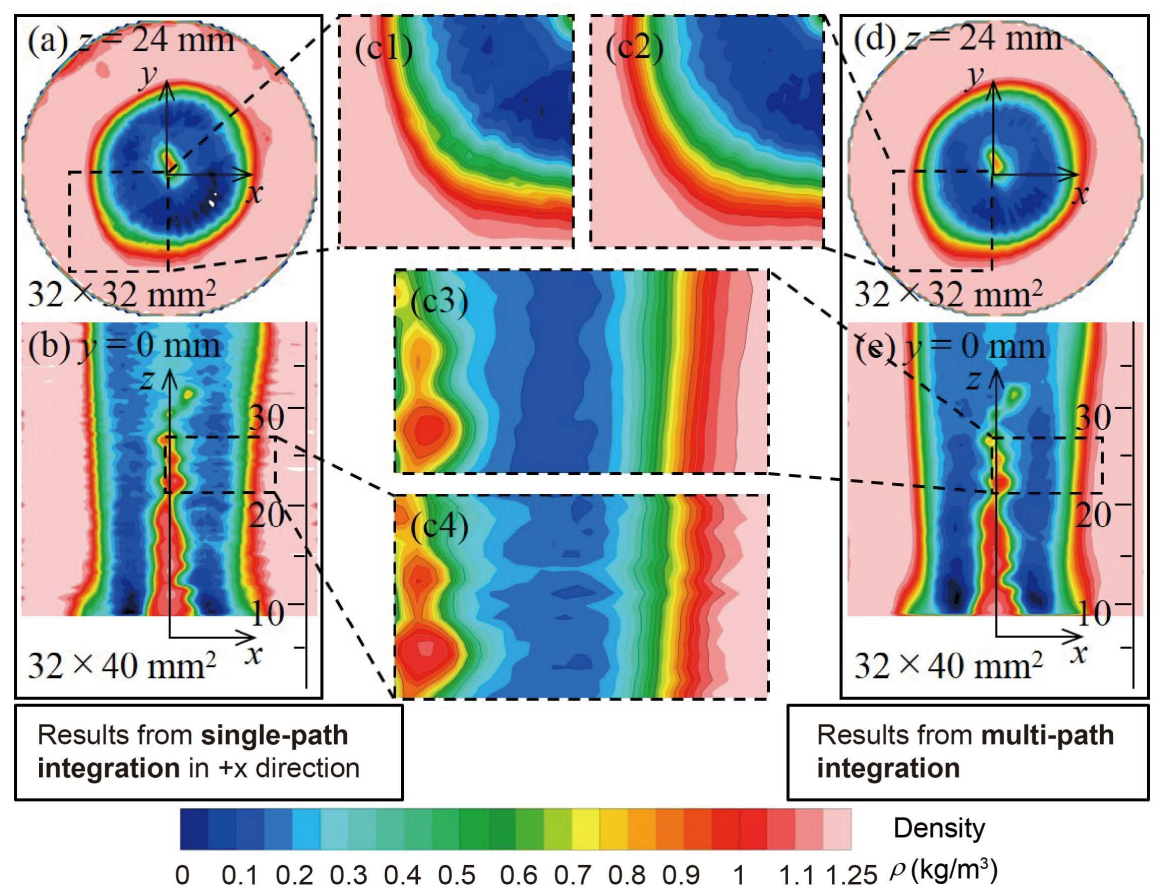

Figure 15. Corresponding density distributions contour diagrams of Figure 14.

—Single-path integration (with noise)

-Multi-path integration (with noise)

- - Multi-path integration (without noise)

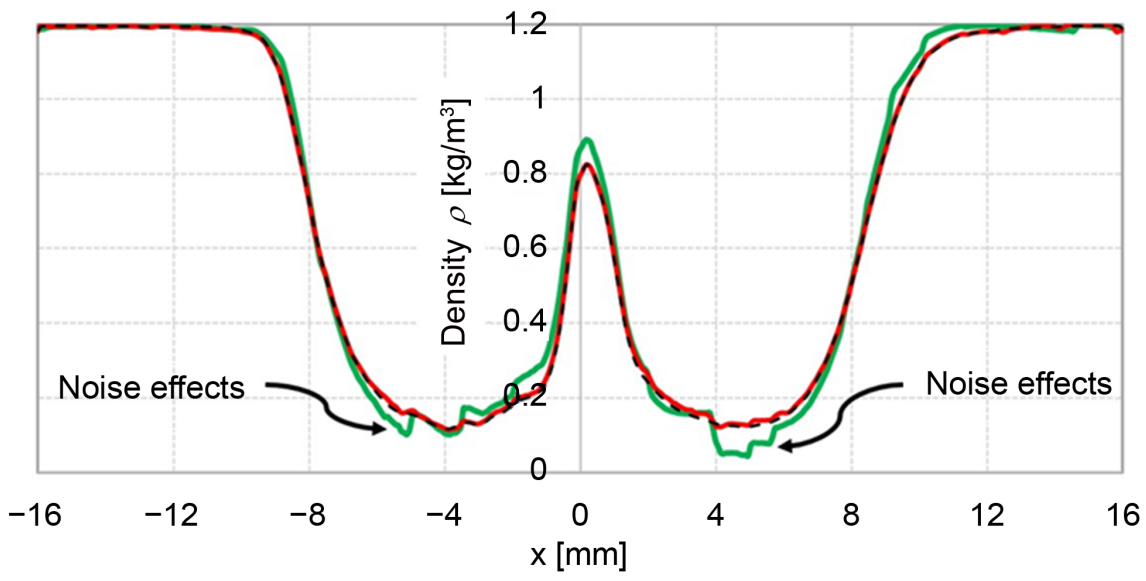

Figure 16. Superposition of radial density distributions of CT-reconstructed density distributions of target flame for $y=0$ and $z=24 \mathrm{~mm}$.

visible on the single-path integration results. Results demonstrate the importance of the new technique for noise reduction.

\section{Conclusions}

We applied the Cauchy integration theorem to develop the noise reduction technique. Based on Cauchy's integration theorem statement, contour integral 
depends only on the beginning and ending points of the paths and does not depend on the integration path. Therefore, the integration procedure in multi-path directions is employed. In the present study, a new technique "multi-path integration" is proposed for "noise reduction" in projections (density thickness) images of CT (computerized tomography). The schlieren brightness gradient information in two orthogonal directions (e.g. horizontal and vertical or two perpendicular diagonal directions) is required in the "multi-path integration" technique. The 20-directional quantitative schlieren optical system gives only images of schlieren brightness in the horizontal gradient and another 20-directional optical system seems necessary to obtain vertical schlieren brightness gradient, simultaneously. One suitable solution without the necessity of a new optical system is using the "inverse process". By employing the new "inverse process" technique, the actual experimental data is used for reproducing other directions of the schlieren brightness gradient. This new approach is a simple, efficient and cost-effective solution and can be used for obtaining any optional directions of schlieren brightness gradient.

In this study, a schlieren image with information on the brightness gradient in two orthogonal directions is called a "complex schlieren image". It has shown an important ability of complex schlieren brightness gradient, which is independence on the path and starting point in the integration process. The results validate that the "multi-path integration" novel technique has the capability of noise reduction in obtaining projections (density thickness) images of CT and the $3 \mathrm{D}$ density distributions of CT results.

\section{Acknowledgements}

This research work was partly supported by The Nitto Foundation and JSPS Grants-in-Aid for Scientific Research (C)16K06118. The supports are gratefully acknowledged.

\section{Conflicts of Interest}

The authors declare no conflicts of interest regarding the publication of this paper.

\section{References}

[1] Settles, G.S. and Hargather, M.J. (2017) A Review of Recent Developments in Schlieren and Shadowgraph Techniques. Measurement Science and Technology, 28 1-25. https://doi.org/10.1088/1361-6501/aa5748

[2] Ishino, Y., Hayashi, N., Bt Abd Razak, I.F. and Saiki, Y. (2015) 3D-CT Measurement of Instantaneous Density Distributions of High-Speed Premixed Turbulent Burner Flames with a Multi-Directional Schlieren Camera (Effects of Flow Velocity on 3D Flame Front Shape). Proceedings of the 1 st Thermal and Fluids Engineering Summer Conference, New York, 9-12 August 2015, 121-131. https://doi.org/10.1615/TFESC1.cbf.012970

[3] Ishino, Y., Hayashi, N., Bt Abd Razak, I.F., Kato, T., Kurimoto, Y. and Saiki, Y. 
(2015) 3D-CT (Computer Tomography) Measurement of an Instantaneous Density Distribution of Turbulent Flames with a Multi-Directional Quantitative Schlieren Camera (Reconstructions of High-Speed Premixed Burner Flames with Different Flow Velocities). Flow, Turbulence and Combustion, 96, 819-835.

https://doi.org/10.1007/s10494-015-9658-5

[4] Ishino, Y., Hayashi, N., Ishiko, Y., Nazari, A.Z., Nagase, K., Kakimoto, K. and Saiki, Y. (2016) Schlieren 3D-CT Reconstruction of Instantaneous Density Distributions of Spark-Ignited Flame Kernels of Fuel-Rich Propane-Air Premixture. Proceedings of ASME Heat Transfer Summer Conference, Washington DC, 10-14 July 2016, HT2016-7423, 1-10. https://doi.org/10.1115/HT2016-7423

[5] Ishino, Y., Hayashi, N., Ishiko, Y., Nagase, K., Kakimoto, K., Nazari, A.Z. and Saiki, Y. (2017) 3D Printing of Spark-Ignited Flame Kernels, Experimentally Captured by 3D-Computer Tomography and Multi-Directional Schlieren Photography. Heat Transfer, 139, HT-16-1726. https://doi.org/10.1115/1.4035583

[6] Nazari, A.Z., Ishino, Y., Motohiro, T., Yamada, R., Ishiko, Y. and Saiki, Y. (2017) Schlieren CT Measurement of 3D Density Distributions of Flame Kernels of Spark-ignited Direct-Injection of Free, Cavity-Guided and Plane-Guided Fuel Jets. Proceedings of the 11th Asia-Pacific Conference on Combustion, Sydney, 10-14 December 2017, 1-4. http://www.anz-combustioninstitute.org/proceedings.php

[7] Nazari, A.Z., Ishino, Y., Ishiko, Y., Ito, F., Kondo, H., Yamada, R., Motohiro, T., Miyazato, Y. and Nakao, S. (2020) Multi-Schlieren CT Measurements of Supersonic Microjets from Circular and Square Micro Nozzles. Journal of Flow Control, Measurement \& Visualization, 8, 77-101. https://doi.org/10.4236/jfcmv.2020.83005

[8] Nazari, A.Z., Ishino, Y., Yamada, R., Motohiro, T., Ito, F., Kondo, H., Miyazato, Y. and Nakao, S. (2018) CT (Computer Tomography) Measurement of 3D Density Distributions of High-Speed Premixed Turbulent Flames (Multi-Path Integration Image-Noise Reduction Technique Based on Novel Concept of Complex Brightness Gradient in Quantitative Schlieren Images). Proceedings of the 18th International Symposium on Flow Visualization, Zurich, 26-29 June 2018, 1-13.

https://doi.org/10.3929/ethz-b-000279202

[9] Nazari, A.Z., Ishino, Y., Yamada, R., Motohiro, T., Ito, F., Kondo, H., Miyazato, Y. and Nakao, S. (2019) CT (Computer Tomography) Measurement of 3D Density Distributions of Flame: Obtaining Vertical Gradient Schlieren Brightness from Horizontal Gradient for Image-Noise Reduction. Proceedings of the 4th Thermal and Fluids Engineering Conference, Las Vegas, NV, 14-17 April 2019, 185-194. https://doi.org/10.1615/TFEC2019.cbf.027746

[10] Huang, C., Gregory, J.W. and Sullivan, J.P. (2007) A Modified Schlieren Technique for Micro Flow Visualization. Measurement Science and Technology, 18, N32-N34. https://doi.org/10.1088/0957-0233/18/5/N04

[11] Collicott, S.H. and Salyer, T.R. (1994) Noise Reduction Properties of a Multiple-Source Schlieren System. AIAA Journal, 32, 1683-1688. https://doi.org/10.2514/3.12160

[12] Chaloupka, J.L., Woods, M., Aas, J., Hutchins, J. and Thistle, J.D. (2014) Color Schlieren Imaging with a Two-Path, Double Knife Edge System. Optics Express, 22, 8041-8046. https://doi.org/10.1364/OE.22.008041

[13] Barry, F.W. and Edelman, G.M. (1948) An Improved Schlieren Apparatus. Journal of the Aeronautical Sciences, 15, 364-365. https://doi.org/10.2514/8.11588

[14] Hadjadj, A. and Kudryavtsev, A. (2005) Computation and Flow Visualization in High-Speed Aerodynamics. Journal of Turbulence, 6, N16. https://doi.org/10.1080/14685240500209775 
[15] Yokoi, T., Shinohara, H., Hashimoto, T., Yamamoto, T. and Niio, Y. (2000) Implementation and Performance Evaluation of Iterative Reconstruction Algorithms in SPECT: A Simulation Study Using EGS4. Proceedings of the Second International Workshop on EGS, KEK Proceedings, 200-20, 224-234.

http://rcwww.kek.jp/egsconf/proceedings/2iwoegs/yokoi.pdf

\section{Nomenclature}

\section{$B$ : Brightness of schlieren image [-]}

$B_{n \dot{ }}$ Brightness of schlieren image in no-flame (no disturbance) condition in the test section [-]

$D t$. Density thickness of deviation density $\left[\left(\mathrm{kg} / \mathrm{m}^{3}\right)(\mathrm{m})\right]$; derived value

$D t$ : Density thickness $\left[\left(\mathrm{kg} / \mathrm{m}^{3}\right)(\mathrm{m})\right]$; derived value

$D t$ : Density thickness of deviation density $\left[\left(\mathrm{kg} / \mathrm{m}^{3}\right)(\mathrm{m})\right]$; actual value

$f$. Focal length of convergent lens in schlieren system [m]

$K$ : Gladstone-Dale constant of air $\left[\mathrm{m}^{3} / \mathrm{kg}\right]$

$R$ : Radius of reconstruction area $[\mathrm{m}]$

$X, y, z$. Cartesian coordinates system of reconstruction volume [m]

$X(\theta), Y(\theta)$ : Inclined coordinates by angle $\theta[\mathrm{m}]$

$\triangle B$ : Deviation brightness on schlieren image [-]

$\Delta s$. Transparent width of the light source $[\mathrm{m}]$

$\Delta \rho$ : Deviation density $\left[\mathrm{kg} / \mathrm{m}^{3}\right]$; derived value

$\Delta \rho^{*}$ : Deviation density $\left[\mathrm{kg} / \mathrm{m}^{3}\right]$; actual value, $\left(\Delta \rho^{*}=\rho_{a}^{*}-\rho^{*}\right)$

$\theta$. Angle of observation [ ${ }^{\circ}$ (degree)]

$\rho$ : Density $\left[\mathrm{kg} / \mathrm{m}^{3}\right]$; derived value, $\left(\rho=\rho_{a}^{*}-\Delta \rho\right)$

$\rho^{*}$ : Density $\left[\mathrm{kg} / \mathrm{m}^{3}\right]$; actual value

$\rho_{a}^{*}$ : Ambient density of air $\left[\mathrm{kg} / \mathrm{m}^{3}\right]$; actual value

$\rho_{\text {re } \dot{i}}$ Reference density $\left[\mathrm{kg} / \mathrm{m}^{3}\right]$

$\varphi$ : Diameter $[\mathrm{m}]$ 